\title{
LA PARADOJA DE LA METAFÍSICA ROMÁNTICA ${ }^{1}$
}

\author{
THE PARADOX OF ROMANTIC METAPHYSICS \\ Frederick C. Beiser
}

\begin{abstract}
RESUMEN
Se presenta, en primer lugar, la metafísica romántica como un intento de fusionar el idealismo fichteano con el realismo spinozista. Se muestran, en segundo lugar, las dificultades encontradas por este intento a la hora de reconciliar las principales afirmaciones de ambas concepciones filosóficas: la creencia de Fichte en la primacía del yo y la fe de Spinoza en la prioridad de la naturaleza.
\end{abstract}

PALABRAS CLAVE: Romanticismo, Fichte, Spinoza, idealismo, realismo, naturalismo, auto-determinación

\begin{abstract}
Romantic Metaphysics is presented as an attempt to fuse Fichtean idealism and Spinozist realism. It is argued that the problem with this attempt is that it not always achieved to reconciliate the main tenents of both idealism and realism: that for the first the self is everything and that for the second it is the world which is everything.

KEYWORDS: Romanticism, Fichte, Spinoza, idealism, realism, naturalism, selfdetermination.
\end{abstract}

\section{UN EXTRAÑO PLAN DE BODA}

A finales de la década de 1790, algunos de los principales pensadores románticos alemanes ya habían esbozado el esquema básico de su nueva metafísica, la Weltanschaunng que más adelante llegaría a ser tan característica de la escuela romántica. De 1795 a 1797, Hölderlin, Novalis y Friedrich Schlegel redactaron

\footnotetext{
${ }^{1}$ El presente texto es una traducción de F. Beiser "The Paradox of Romantic Metaphysics", en N. Kompridis (ed.) Philosophical Romanticism, New York: Routledge, 2006, pp. 217-237. Traducido con permiso del autor. Traducción española a cargo de David Pérez Chico.
} 
los rudimentos de esta metafísica en distintos fragmentos; con posterioridad, Schelling ofreció una formulación más sistemática en su System des transcendentalen Idealismus (1890) y en su Darstellung meines Systems (1891). Existen algunas diferencias importantes en la formulación de esta doctrina por parte de cada uno de estos pensadores; pero también encontramos algunas similitudes asombrosas, algunas características compartidas. Será precisamente una de estas características nuestra principal preocupación.

Se trata del intento por parte de la metafísica romántica de fusionar el idealismo y el realismo y, más específicamente, el idealismo de Fichte y el realismo de Spinoza. ${ }^{2}$ Esta característica es, prima facie, desconcertante y problemática. Sin duda, Fichte y Spinoza fueron los filósofos que más influyeron en la generación romántica. Pero también eran, en un sentido fundamental, completamente incompatibles: el idealismo, el indeterminismo y el dualismo de Fichte chocan de frente con el realismo, el determinismo y el monismo de Spinoza. Aunque eran perfectamente conocedores de estas incompatibilidades, lo románticos insistieron en querer unirlos. No les servía otra cosa que no fuera esta unión porque, a su modo de ver, cada uno había capturado únicamente una mitad de la verdad. De igual manera que las parejas de novios perfectas, Fichte y Spinoza eran los complementos perfectos en un todo indisoluble.

${ }^{2}$ La evidencia de que los románticos tenían un proyecto de esta índole es palmaria en sus cuadernos y fragmentos. En el caso de Schelling, véase su Briefe über Dogmatismus und Kriticismus, Werke I, 326-35. El idealismo absoluto tardío de Schelling, tal y como queda expresado en el System des transcendentalen Idealismus y en Darstellung meines Systems, puede ser visto como el intento de sintetizar el idealismo de Fichte y el realismo de Spinoza. En el caso de Novalis, véase, por ejemplo, Allgemeine Brouillon, \#75, 634, 820; HKA III, 252, 382, 429; y Fragmente und Studien \#611; HKA III, 671. En el caso de Friedrich Schlegel, véase, por ejemplo, Philosophische Lehrjahre. KA XVIII, 31, 38, 43, 80. En el caso de Hölderlin, véase el prefacio de la penúltima versión de Hyperion, GSA III/1, 236; el prefacio de Fragment von Hyperion, III/1, 163; y la versión final de Hyperion, III/1, 38. El intento de unir a Fichte y a Spinoza era uno de los principales objetivos de la temprana novela de Hölderlin. Este proyecto fue probablemente tema de discusión entre Hölderlin y Schelling alrededor de 1796-7. Véase las Briefe über Dogmatismus und Kriticismus, Werke I, 284-90, de Schelling. En la penúltima carta de las Briefe Schelling esboza su propia idea de lo que sería la síntesis de idealismo y realismo, idea que más adelante se concretó en su Identitätssystem. Véase Werke I, 326-30. Es imposible determinar quién de entre los románticos fue el impulsor original de este proyecto porque lo concibieron aproximadamente al mismo tiempo, en parte independientemente, en parte conjuntamente en sus conversaciones, en especial durante los años de formación 1796-98. 
La primera reacción que muy probablemente pueda tener alguien en relación con este proyecto es la de pensar que se trata de un absurdo quijotesco, otra Schnappside romántica que, afortunadamente, nunca llegó a fructificar. El idealismo fichteano y el realismo spinoziano son tan contradictorios, tan claramente opuestos tanto en propósito como en contenido, que da la sensación de que cualquier intento de unirlos está abocado al fracaso. Uno podría igualmente intentar cuadrar el círculo. Sin duda, no es una buena señal que Kant y el propio Fichte descartaran cualquier intento de unión con Spinoza. Explícita y enfáticamente concibieron su propio idealismo como la antitesis del realismo y del naturalismo spinozianos. ${ }^{3} \mathrm{El}$ único remedio contra este fatalismo, nos advirtieron, se encuentra en su idealismo trascendental.

Semejante desconfianza plantea algunas preguntas. ¿De qué manera intentaron los románticos unir a Fichte y a Spinoza? Y, ¿podría semejante síntesis ser coherente? Cualquier intento de comprender la Weltanschanung romántica tiene, en mi opinión, que dar respuesta en algún momento a estas cuestiones. Demasiado a menudo la metafísica romántica es entendida como una forma poética del idealismo fichteano o del naturalismo spinoziano, como si fuera lo uno o lo otro. Pero estas interpretaciones comunes son demasiado unilaterales, y no tienen en cuenta la característica más sorprendente de la metafísica romántica: su intento de esposar a Fichte y a Spinoza. Si no se entiende este matrimonio, el rasgo más característico de la metafísica romántica seguirá siendo un enigma, una paradoja. Aunque pueda parecer un proyecto caducado, el intento de fusionar a Fichte y a Spinoza contiene aún hoy algo de interés. Hoy en día los filósofos se refieren a menudo a la bancarrota de la tradición cartesiana, cuya epistemología conduce a un subjetivismo absoluto: la limitación del conocimiento al círculo de la conciencia; estos filósofos encuentran el antídoto a este subjetivismo en una ontología naturalista, o heideggeriana, que convierte al yo o bien en parte de la naturaleza o bien en parte de la historia. Pero semejante remedio es problemático, entre otras razones porque no resuelve los problemas escépticos que motivaron originariamente la epistemología cartesiana: ¿Cómo sabemos que existe una naturaleza o una historia más allá de la conciencia? Se nos deja, así pues, con un dilema: o una epistemología escéptica o una ontología dogmática. Uno de los aspectos más fascinantes de la metafísica romántica es que intentó superar estos extremos. Los jóvenes románticos eran perfectos conocedores de este dilema:

\footnotetext{
${ }^{3}$ Véase Fichte, Grundlage dergesammten Wissenschaftslebre, Werke I, 101; y Kant, Kritik derpraktischen Vernunft, AA V, 101-2.
} 
lo tenían presente a la hora de elegir entre la crítica kantiana y el dogmatismo spinoziano. El objetivo de su intento de fusionar a Fichte y a Spinoza era superar este dilema evitando los extremos tanto del subjetivismo como del dogmatismo, y combinando las virtudes de una epistemología crítica y las de una ontología naturalista.

Lo que sigue es un intento de desentrañar la paradoja de la metafísica romántica, de explicar su intento de superar este dilema aparentemente eterno. Defenderé que la metafísica romántica es perfectamente comprensible y coherente siempre que la situemos en el contexto de su concepto orgánico subyacente de naturaleza. Este concepto fue el que permitió a los románticos unir el idealismo fichteano con el naturalismo spinoziano; la creencia de Fichte en la primacía del yo y la fe de Spinoza en la prioridad de la naturaleza.

Por supuesto, no es ninguna novedad que se enfatice la importancia que el concepto orgánico tiene para el romanticismo. A lo largo de generaciones, los especialistas han subrayado su papel crucial, viéndolo como una de las características centrales de la Weltanschaunng romántica. ${ }^{4}$ No obstante, debemos decir que esta visión tradicional no concuerda con la tendencia más reciente de Romantikforschung, que ha cuestionado el viejo énfasis en el concepto orgánico de naturaleza, y que ha subrayado en su lugar la ausencia de completitud y de clausura en el pensamiento romántico. ${ }^{5}$ No obstante, en breve veremos que este concepto orgánico es indispensable a la hora de desentrañar la paradoja de la metafísica romántica y, más específicamente, a la hora de comprender su intento aparentemente quijotesco de esposar el idealismo con el realismo. Esto ayudará a vindicar la vieja tradición académica y a devolver al lugar que le corresponde a lo orgánico en el pensamiento romántico.

\footnotetext{
${ }^{4}$ Véase, por ejemplo, Alois Stockman, Die deutsche Romantik, Freiburg, Herder, 1921, pp. 1317; Oskar Walzel, "Wesenfragen deutscher Romantik," Jahrbuch des Freien deutschen Hochstifts 29 (1929), 253-76; Adolf Grimme, Vom Wesen der Romantik, Braunschweig, Westermann, 1947, p. 13; René Wellek, "The Concept of Romanticism in Literary History" y "Romanticism Reexamined," en Concepts of Criticism, ed. Stephen G. Nichols, New Haven, Yale University Press, 1963, pp. 165, 220; More Peckham, "Toward A Theory of Romanticism," en Proceedings of the Modern Language Association 66 (1951), 5-23; y Lawrence Ryan, "Romanticism," en Periods of German Literature, J. M. Ritchie (ed.), Londres, Oswald Wolff, 1966, pp. 123-43.

${ }^{5}$ Véase, por ejemplo, Paul de Man, "The Rhetoric of Temporality," en Blindness and Insight, 2nd edn,. Minneapolis, University of Minnesota Press, 1983, pp. 187-228, esp. 220-8; y Alice Kuzniar, delayed Endings: Nonclosure in Novalis and Hölderlin, Athens GA, University of Georgia Press, 1987, pp. 1-71.
} 


\section{PERFIL DE UN DESAJUSTE}

Antes de que expliquemos el proyecto romántico es necesario tener una idea más concreta del desafío que tienen ante sí. Más exactamente, ¿cuáles eran las incompatibilidades entre Fichte y Spinoza?

Existen algunas cuestiones básicas que separan a Fichte y a Spinoza. En primer lugar, están en desacuerdo con respecto a la realidad del mundo externo o de la naturaleza. La filosofía de Fichte es idealista: niega la realidad de la cosa en sí, esto es, cualquier cosa que exista con independencia del conocimiento que podamos tener de ella; y sostiene que todo existe gracias a una conciencia real o posible. En cambio, la filosofía de Spinoza es realista: afirma la realidad de la naturaleza al completo, que existe con independencia y con anterioridad a cualquier conocimiento de la misma. En lugar de existir únicamente para un determinado sujeto, la subjetividad es simplemente un modo, apariencia, o parte de la naturaleza al completo. En segundo lugar, Fichte y Spinoza chocan frontalmente con respecto al alcance de la explicación natural. La filosofía de Fichte es anti-naturalista: limita el ámbito de la naturaleza a la experiencia, y postula la existencia de un ámbito de la razón y la libertad más allá de la experiencia. La filosofía de Spinoza es, en cambio, radicalmente naturalista: lo sitúa todo en el interior de la naturaleza, de tal manera que nada escapa a sus leyes. A partir de esta segunda diferencia Fichte afirma, y Spinoza niega, la realidad de la libertad humana en un sentido muy importante: la libertad de elección, la capacidad para hacer las cosas de otra manera independientemente de las causas externas. Para Spinoza la volición y la acción humanas forman parte de la naturaleza, de ahí que ocurran con necesidad siguiendo sus leyes; para Fichte, en cambio, la volición y acción humanas trascienden la naturaleza, de tal manera que es posible que sean de otra manera.

El conflicto entre Fichte y Spinoza alcanza sus extremos cuando se tiene en consideración la tercera cuestión que los mantiene separados: sus visiones opuestas de la realidad última o lo absoluto. Fichte convierte el ego en su absoluto, proponiéndose explicar toda la naturaleza como un producto de aquél; Spinoza convierte a la naturaleza en su absoluto e intenta explicar el ego como producto de la misma. No podemos, por lo tanto, combinar a Fichte y a Spinoza, como tampoco podemos afirmar la existencia de dos absolutos, de dos realidades infinitas. No podemos simultáneamente "hacer que el yo sea todo y el mundo nada $y$ que el mundo sea todo y el yo nada."

\footnotetext{
${ }^{6}$ Esta es la formulación de Hölderlin en el prefacio de la penúltima versión de Hyperion, GSA III/1, 236.
} 
Nuestras sospechas acerca de la síntesis romántica no hacen más que aumentar cuando valoramos lo que los románticos vieron en Fichte y en Spinoza. Lo que atrajo de Fichte a los románticos fue su concepto radical de libertad humana, según la cual el yo se pone a sí mismo, se hace a sí mismo. Fue este concepto del yo que se pone a sí mismo el que racionalizó la Revolución Francesa, el derecho del yo para rehacer leyes e instituciones de acuerdo con las exigencias de la razón. En tanto simpatizantes de la Revolución en Francia, los jóvenes románticos no podían sino abrazar el concepto que anidaba tras ella. Lo que los románticos admiraban de Spinoza era su síntesis de la religión y la ciencia. Parecía que el panteísmo spinoziano resolvía todos los conflictos tradicionales entre la razón y la fe. Había creado una religión a partir de la ciencia al divinizar la naturaleza, y una ciencia a partir de la religión al naturalizar lo divino. Pero es precisamente en estos aspectos donde Fichte y Spinoza parecen completamente irreconciliables. Si la naturaleza es divina, entonces es infinita, y todo debería obedecer sus leyes; por lo tanto no puede haber ningún ámbito trascendental de libertad por encima y más allá de la naturaleza. En lugar de crearse a sí mismo, el yo simplemente realizará necesariamente la esencia que le ha sido dada por el orden natural de las cosas.

Dadas todas estas incompatibilidades, parece que no queda esperanza para un matrimonio entre Fichte y Spinoza. No podemos esposarlos como tampoco podemos emparejar a idealistas con realistas, ni a dualistas con monistas, ni a indeterministas con deterministas. Si en ocasiones se dan matrimonios entre opuestos, se debe únicamente a alguna afinidad subyacente más profunda. Pero aquí no encontramos ninguna. ¿Por qué intentar unir a enemigos?

\section{IDENTIDAD SUJETO-OBJETO}

Irónicamente, fue Fichte el que inspiró en primer lugar los esfuerzos por parte de los románticos de establecer estos contactos. A pesar de toda su bravuconería contra Spinoza, Fichte virtualmente forzó a los románticos a abrazar su némesis como si de su complemento necesario se tratara. Esto fue el inevitable resultado de uno de los principios fundamentales del Wissenschaftslehre, el principio al que Fichte en ocasiones denominó identidad sujeto-objeto, la tesis de que lo subjetivo y lo objetivo, lo ideal y lo real, son en última instancia lo mismo. Los románticos se basaron en este principio para racionalizar su intento de llegar más lejos que Fichte. Para ellos el polo subjetivo o ideal refleja el idealismo fichteano, el polo objetivo o real representa el realismo spinoziano. 
¿Por qué se sintieron los románticos obligados a interpretar el principio de Fichte de una manera tan anti-fichteana? La respuesta reside en las dificultades inherentes a la interpretación del propio Fichte de su principio. En la Wissenschaftslebre de Jena Fichte interpretó la identidad sujeto-objeto en términos de auto-conocimiento. Dado que conocedor y conocido son lo mismo en el auto-conocimiento, este posee la identidad entre sujeto y objeto, ideal y real, requerida. Fichte creía que este hecho por sí solo debería convertir al auto-conocimiento en el paradigma de todo conocimiento. Como quiera que todo conocimiento presupone alguna identidad entre conocedor y conocido, y dado que tal identidad es demostrable en el caso del auto-conocimiento, el auto-conocimiento debería ser la base de todo conocimiento. Si podemos mostrar que, de alguna manera, todo conocimiento es una forma de auto-conocimiento — aunque sea auto-conocimiento subconsciente- entonces le habremos proporcionado un fundamento. ${ }^{7}$

La inspiración tras el principio de Fichte era nada menos que la idea rectora tras el "nuevo método de pensamiento" de Kant: solo conocemos a priori aquello que creamos. ${ }^{8}$ Kant había afirmado que la actividad innata de la mente es transparente para sí misma, siendo así que conoce cualquier cosa que crea. ${ }^{9}$ Dado que el yo incorpora o revela su actividad en sus objetos, su conocimiento de los mismos equivale a una forma de auto-conocimiento, siendo así que el auto-conocimiento es el paradigma de todo conocimiento. Este paradigma de conocimiento desempeña un papel básico — si bien no enteramente explícito- en la Deducción Trascendental de la primera Kritik en la forma de la unidad de apercepción, el "yo pienso" o autoconsciencia que acompaña a todas sus representaciones. Semejante auto-consciencia equivale a la consciencia por parte del yo de su propia creatividad, cuyos productos son las distintas formas de una síntesis a priori, a saber, las categorías del entendimiento y las formas de la sensibilidad: espacio y tiempo.

Los románticos adoptaron, en ciertos aspectos fundamentales, el principio fichteano de la identidad sujeto-objeto. Estaban de acuerdo con Fichte en que el principio de la identidad sujeto-objeto equivale a alguna forma de autoconocimiento, y en que el auto-conocimiento debe ser la base de todo conocimiento. Pero discreparon con él respecto a su lectura unilateral subjetivista de este principio.

\footnotetext{
${ }^{7}$ Este es el razonamiento de Schelling en su temprano trabajo fichteano Abhandlungen zur Erläuterung des Idealismus der Wissenschaftslehre, Werke I, 101.

${ }^{8}$ Véase Kant, Kritik der reinen Vernunft, Vorrede, B xviii.

${ }^{9}$ Ibid., B xiii, A xx, A xiv.
} 
La lectura que hace Fichte de este principio es, en su opinión, subjetivista en la medida en que localiza la identidad sujeto-objeto en la autoconsciencia del sujeto trascendental, en el Ich denke de la unidad de la apercepción o el Ich bin Ich de la Wissenschaftslehre. Para Fichte, la identidad sujeto-objeto esencialmente consiste en un auto-conocimiento trascendental, la consciencia del sujeto de su actividad espontanea. Insistió en que esta identidad debe ser inmanente, situada dentro del ámbito de la consciencia posible; localizarla fuera de este ámbito, como hizo Spinoza, fue trascendente, nada menos que una hipóstasis, "la falacia de la razón pura." 10

Los románticos realizaron dos objeciones fundamentales contra la interpretación subjetivista fichteana del principio de la identidad sujeto-objeto. En primer lugar, los conceptos mismos de lo subjetivo y lo objetivo solo tienen sentido en oposición mutua; poseen sus significados específicos únicamente en el ámbito de la experiencia; pero el principio es supuestamente trascendental, y explica la posibilidad misma de la experiencia y por lo tanto no puede estar presente en la experiencia; en ese caso, por tanto, no puede ser ni subjetivo ni objetivo. ${ }^{11}$ En segundo lugar, semejante lectura impide que el principio realice su función consistente en explicar la posibilidad de conocimiento. Si es un principio constitutivo o teorético, tal como "yo soy" o "yo pienso", entonces no puede extraer de la experiencia el dualismo entre sujeto y objeto, ni tampoco el contenido del conocimiento empírico. Si, por el contrario, se trata de un principio regulativo o práctico que no expresa sino el esfuerzo del ego para controlar la naturaleza, entonces nos vemos atrapados en un dilema. En la medida en que el ego domine la naturaleza, el objeto no es nada más que un producto de su actividad, un mero noúmeno; pero si no domina la naturaleza, el objeto es incognoscible, una X. Tenemos, por lo tanto, que elegir entre auto-conocimiento y una cosa-en-sí. Hacia el final del Wissenschaftslehre de 1794, Fichte admite que este dilema es inevitable para un conocedor finito. Para los jóvenes Schelling y Hegel esto equivale a admitir su fracaso. ${ }^{12}$

${ }^{10}$ Fichte, Grundlage der gesamten Wissenschaftslebre, Werke I, 101.

${ }^{11}$ Esta crítica es más explícita en el famoso fragmento de Hölderlin "Urtheil und Seyn,” GSA IV/1, 216-17. Pero Hölderlin no es el único que la hizo. Schelling defendió lo mismo en Briefe über Dogmatismus und Kriticizismus, Werke I, 329; y Vom Ich als Princip del Philosophie, Werke I, 180-1. Véase también Novalis, Fichte Studien, HKA II, 107; \#5-7.

${ }^{12}$ Esta segunda crítica resulta más evidente en el primer Hegel y en Schelling. Véase su Fernere Darstellung aus dem System del Pbilosophie, en Schelling, Werke IV, 356-61; y su "Ueber das Verhältniß der Naturphilosophie überhaupt," en Schelling, Werke V, 108-15. 
La esencia de la crítica romántica al principio de Fichte es que no le hace justicia a la realidad de la experiencia, a la existencia del mundo externo. El principio de Fichte de la identidad sujeto-objeto es subjetivista porque no acomoda la experiencia del mundo objetivo. Prima facie esta objeción da la impresión de ser injusta porque Fichte pretendía que su idealismo trascendental fuese una forma de realismo empírico, que es lo que explica la realidad de las cosas en el espacio con independencia del sujeto consciente. De hecho, el propósito principal de la Wissenschaftslehre era explicar el sentimiento de necesidad que acompaña a nuestras representaciones, el hecho de que parece que vienen y van con independencia de nuestra voluntad e imaginación. Pero los románticos eran perfectamente conscientes de las intenciones de Fichte; su crítica contra éste es doble: en primer lugar el realismo empírico es insuficiente; y en segundo lugar, Fichte ni siquiera lo garantiza. El realismo empírico es insuficiente porque aunque permite que los objetos existan con independencia del yo empírico, no les permite existir con independencia del yo trascendental, que es el legislador de la naturaleza. Los románticos querían llegar más lejos que Fichte al otorgar una realidad independiente a la naturaleza; demandaban un "realismo superior", el cual otorgaría realidad a la naturaleza con independencia del yo, ya fuera empírico o trascendental. ${ }^{13}$ Este realismo superior era tanto una razón para, como el resultado de, su simpatía por Spinoza. En segundo lugar, Fichte no pudo ni tan siquiera establecer su realismo empírico porque admitió que el primer principio de la Wissenschaftslebre, "el yo se pone absolutamente", no puede derivar la realidad del no-yo, el cual se opone a sí mismo. Todo el inmensamente sutil y sofisticado razonamiento de la primera parte de la Grundlage der gesammten Wissenchaftslebre de 1794 llega finalmente a la conclusión de que el ego que se opone a sí mismo no se puede limitar a sí mismo poniendo un no-yo contrario a sí mismo. El fracaso del programa de deducción fichteano en la Grundlage de 1794 fue clave para convencer a los románticos de superar los límites de sus propios principios subjetivistas.

\section{EL CONCEPTO ORGÁNICO DE NATURALEZA}

Fue la necesidad de explicar la realidad del mundo externo, de hacer justicia a la pura otredad del no-yo, lo que eventualmente empujó a los románticos a

\footnotetext{
${ }^{13}$ Para Schlegel, véase, por ejemplo, Philosophische Lehrjahr, KA XVIII, 31 (\#134), 38 (\#209), 80 (606). Sobre Novalis, véase Allgemeine Brouillon, HKA III, 382-4 (\#634), 252 (\#69), 382 (\#633), 429 (\#820); y Fragmente und Studien, HKA III, 671 (\#611).
} 
abandonar el unilateralismo del idealismo fichteano y a complementarlo con el "realismo superior" de Spinoza. De una u otra forma iban a tener que encontrar una interpretación del principio de la identidad sujeto-objeto que diera acomodo a nuestra experiencia de un mundo externo. Se trataba de una iniciativa inherentemente paradójica ya que el principio de la identidad sujeto-objeto postula la identidad de sujeto y objeto, pero la experiencia ordinaria parece que nos muestra que son el uno distinto del otro. De una u otra forma, tendría que haber una identidad entre la identidad sujeto-objeto y la no-identidad sujeto-objeto. Pero con la mera formulación del deseo uno parece contradecirse a sí mismo. Otra manera de formular este deseo — no menos paradójica que la anterior- consiste en afirmar que debe existir alguna clase de identidad entre el idealismo fichteano y el realismo spinoziano.

Para los románticos, la manera de salir de este atolladero reside en su concepción orgánica de la naturaleza. Esta concepción fue desarrollada con mayor detalle y rigor por Schelling, primero en su Von der Weltseele escrito en 1799, y en su Entwurf eines Systems der Naturphilosophie, de 1798. Pero la misma idea aparece también en los cuadernos de notas de Friedrich Schlegel, Novalis y Hölderlin.

El concepto fundamental tras el concepto romántico de naturaleza es el de fin natural (Naturzweck). Kant ha definido de manera muy específica este concepto en la sección 65 de la Kritik der Urteilskeraft, un texto crucial para Schelling y para los románticos. Algo es un propósito natural, escribió Kant, únicamente si satisface dos condiciones esenciales. En primer lugar, debe poseer una unidad orgánica, donde cada parte es inseparable del todo, y donde la idea del todo determina el lugar que le corresponde a cada parte. En segundo lugar, debe ser auto-generador y auto-organizador, de tal manera que todas las partes son recíprocamente la causa y el efecto las unas de las otras, sin que exista una causa externa. Kant defiende que esta segunda condición es la característica específica de un propósito natural en lugar de una obra de arte. Las obras de arte y los propósitos naturales tienen en común que son producidos siguiendo una cierta del todo; pero únicamente un propósito natural es auto-productor. Su diseño y estructura surgen desde dentro de acuerdo con algún principio interno; no surgen de fuera, como es el caso de la obra de arte.

El concepto orgánico de naturaleza surgió de la generalización, o extensión, de la idea kantiana de fin natural, de tal manera que se aplicaba a la naturaleza en su conjunto. El concepto orgánico significa que la naturaleza al completo es un vasto fin natural, cada una de cuyas partes también es un fin similar, siendo que la naturaleza es un organismo de organismos. Este concepto postula una única 
fuerza vital a lo largo y ancho de toda la naturaleza, de tal manera que las diferentes especies de minerales, plantas y animales, e incluso todas las diferentes clases de materia, son simplemente otros tantos grados diferentes de su organización y de su desarrollo. Toda la naturaleza, por lo tanto, constituye una enorme jerarquía, que consiste en las distintas etapas de organización y desarrollo de la fuerza vital. La fuerza vital se manifiesta primero en las formas más simples de materia; a continuación pasa por los minerales más complejos, por los vegetales, y por los animales; y finalmente alcanza las formas más sofisticadas de vida, tales como la auto-consciencia del filósofo trascendental y la creatividad del propio genio artístico. Semejante auto-consciencia es nada menos que la organización y el desarrollo supremos de todas las potencias de la naturaleza. Esto quiere decir que la conciencia que tiene el artista o el filósofo de la naturaleza es, además, la propia naturaleza adquiriendo auto-consciencia a través de ellos.

La implicación más importante del concepto orgánico de naturaleza es que no hay distinción de clase, sino únicamente de grado, entre lo mental y lo físico. Lo mental y lo físico ya no son sustancias heterogéneas, sino que son únicamente diferentes niveles de organización y desarrollo de la fuerza vital única a lo largo y ancho de la naturaleza. Lo mental es simplemente el grado superior de organización y desarrollo de las fuerzas vitales activas en la materia; y la materia es meramente el grado inferior de organización y desarrollo de las fuerzas vitales presentes en la mente. Podemos, por lo tanto, considerar que la mente es materia sumamente organizada y desarrollada, y que la materia es una mente menos organizada y desarrollada. Como lo expuso Schelling: "La naturaleza debe ser espíritu visible, y el espíritu naturaleza invisible” (II 56)

\section{EL FUNDAMENTO DEL ORGANICISMO}

No cabe duda de que el concepto orgánico de naturaleza de Schelling es arriesgado e imaginativo, $\mathrm{y}$ ha sido adecuadamente criticado por positivistas y neokantianos por ser descuidado y excesivamente especulativo. Pero tales juicios no tienen en cuenta el contexto de la Naturphilosophie, y más específicamente la crisis de la física y la fisiología a finales del siglo XVIII. Aunque Schelling no podía - y no lo hizo- presumir de una prueba experimental definitiva de su concepto, consideraba que estaba garantizado por los últimos resultados científicos de su época. Más importante, sin embargo, es que Schelling desarrolló su concepto porque consideraba que se trataba de la única solución a los persistentes problemas de la fisiología y la física. El principal argumento a favor del concepto orgánico era 
más conceptual que empírico: parecía resolver por sí solo las crisis inherentes a la ciencia natural del siglo XVIII.

Schelling propuso este concepto en primer lugar y ante todo como solución a un dilema aparentemente ineludible que venía preocupando a la fisiología desde principios del siglo XVII: dualismo versus materialismo mecanicista. Estos extremos parecían ser las únicas posibilidades si se adoptaba el concepto cartesiano de materia así como su paradigma de explicación mecanicista. Según este concepto, la esencia de la materia es su extensión, y es inherentemente inerte, moviéndose únicamente si es movida por alguna fuerza externa. Según este paradigma, explicamos todos los eventos como si se debieran al impacto de un cuerpo sobre otro, donde medimos el impacto en función de cuánto debe cambiar un cuerpo su posición en un intervalo de tiempo dado. Si adoptamos este concepto y este paradigma, entonces tenemos que elegir entre dos alternativas no deseadas: o bien situamos la vida fuera de la naturaleza - y nos convertimos en dualistas - o la reducimos a materia en movimiento $-\mathrm{y}$ nos convertimos en mecanicistasPero ambas alternativas son insatisfactorias. Si bien el mecanicista mantiene los principios del naturalismo, parece ignorar las cualidades características de la vida; y aunque el dualista reconoce tales cualidades, las transporta a un misterioso dominio sui generis en el que no pueden ser explicadas según los métodos de la ciencia.

El propósito del concepto orgánico de Schelling era proporcionar un camino intermedio entre los cuernos de este dilema. ${ }^{14}$ Schelling estaba de acuerdo con el dualista en que el mecanicismo no podía explicar las características sui generis de la vida; pero al mismo tiempo simpatizaba con los esfuerzos del materialista para explicar la vida en base a leyes naturales. En su opinión, únicamente el concepto orgánico de naturaleza podría evitar los problemas tanto del dualismo como del materialismo al proporcionar una concepción naturalista y no reductivista de la vida y de la mente. Como un organismo no es reducible a un mecanismo, no reduce la vida a una máquina; pero como además actúa siguiendo las leyes naturales, no se produce ninguna violación de los principios del naturalismo. Por lo tanto, el concepto orgánico pone en tela de juicio la premisa compartida por el dualismo y el materialismo: que toda explicación natural es mecánica. En lugar de dar cuenta de eventos naturales a partir de causas externas que actúan sobre ellos, los explica en base al lugar necesario que ocupan en un todo sistemático. El paradigma explicativo pasa a ser holista en lugar de analítico o atomista.

${ }^{14}$ Véase Schelling, Von del Weltseele, Werke II, 496-505; y su Erster Entwurf eines Systems der Naturphilosophie, Werke III, 74-8. 
El concepto orgánico de Schelling no sólo fue dictado por una crisis en fisiología, sino también por una en física. A finales del siglo XVIII el principal desafío para la física provenía esencialmente del hecho de que el paradigma mecanicista, que había dominado la física desde Descartes, ya no parecía dar cuenta ni siquiera de la materia. La raíz del problema residía en la ley de la gravedad de Newton. Nadie podía dudar de la ley de Newton, pues había sido confirmada una y otra vez por medio de la observación y la experimentación; sin embargo postulaba una fuerza de atracción entre los cuerpos que aparentemente operaba en el espacio vacío. De ahí surgió la cuestión de la "acción a distancia". Esto constituía un serio problema para la física mecanicista, que insistía en que un cuerpo actúa sobre otro únicamente por medio de un impacto, un cuerpo golpeando a otro. Obstinada pero desesperadamente, algunos físicos trataron de explicar la atracción gravitatoria postulando para ello la existencia de fluidos o sustancias sutiles entre los cuerpos; pero los experimentos fracasaron a la hora de detectar su presencia. ${ }^{15}$

A finales del siglo XVIII daba la impresión de que la investigación sobre la electricidad, el magnetismo y la química supondría el golpe de gracia al mecanicismo. El problema de explicar la acción a distancia pasó a ser todavía más importante porque los últimos descubrimientos en electricidad y en magnetismo sugerían que la mismísima materia consistía en fuerzas de atracción y repulsión; parecía como si las fuerzas que Newton había postulado para el macrocosmos también fueran válidas para el microcosmos. Ya no parecía que la esencia de la materia fuese la extensión inerte, sino la fuerza dinámica. Pero si esto es así, entonces no es sólo la vida sino también la materia la que se resiste a ser explicada mecánicamente. Esta, en cualquier caso, fue la principal conclusión del primer trabajo de Schelling sobre Naturphilosopbie, su Ideen zu einer Pbilosopbie der Natur de 1797.

En la actualidad ocupamos una posición mucho mejor para entender las causas intelectuales generales que empujaban a Schelling hacia su concepto orgánico de naturaleza. Si era necesario ampliar la visión del mundo naturalista, de tal manera que la mente y la vida sean parte de la naturaleza; y si, además, no podía darse una explicación mecánica de la materia, mucho menos de la vida y de la mente; entonces el único paso adelante consistía en ampliar el paradigma organicista de tal manera que fuese válido para toda la naturaleza sin excepción. La gran promesa del paradigma organicista consiste en que, con posterioridad al

\footnotetext{
15 Sobre estos experimentos, véase Thomas Hankins, Science and Enlightenment, Cambridge, Cambridge University Press, 1992, pp. 46-80.
} 
colapso del mecanicismo, garantizaba el principio de la unidad de la naturaleza, una única forma de explicación tanto para la vida como para la materia. La lex continui fue finalmente confirmada. Mente y materia, lo orgánico y lo inorgánico, ya no estaban separadas, sino que simplemente eran manifestaciones diferentes de la fuerza vital.

El gran predecesor de este concepto orgánico de naturaleza era aquel viejo Erajfeind del cartesianismo: Leibniz. No el Leibniz exotérico de la armonía preestablecida para el que lo mental y lo físico constituían ámbitos distintos, sino el Leibniz esotérico de la monadología, para el que la materia era solo una apariencia de la fuerza vital. No es casualidad que Herder y Schelling, consciente y explícitamente, revivieran a Leibniz. ${ }^{16}$ Irónicamente el archidogmático que recientemente había sido enterrado por Kant, había sido resucitado. El momento de Leibniz finalmente había llegado; a pesar de su peluca barroca, se había convertido en un favorito de la época romántica.

\section{SPINOZISMO REVITALIZADO}

Naturalmente, otro autor querido fue "el sagrado Spinoza", a quien Schlegel, Novalis, Schelling, Hölderlin y Schleiermacher rendirían homenaje. Más adelante Hegel diría que uno tenía que aprender a filosofar en el éter de la substancia spinoziana, resumiendo así la convicción de una generación entera. ${ }^{17}$ Sin embargo, sería un grave error pensar que la metafísica romántica no es nada más que una revivificación de Spinoza. Porque los románticos re-interpretaron exhaustivamente a Spinoza, de hecho, lo hicieron de un modo que hubiera provocado que Benedictus se revolviera en su tumba. Pues por supuesto, únicamente podían integrar su naturalismo y su realismo con el idealismo de Kant y Fichte reintepretando a Spinoza.

Los románticos se sintieron especialmente atraídos por dos aspectos del sistema spinoziano. En primer lugar su monismo, su creencia en que hay un único universo del cual lo mental y lo físico son tan solo diferentes atributos. El monismo spinoziano era la antítesis del legado dualista de la tradición cartesiana, la misma que tantos problemas había creado en fisiología. En segundo lugar su panteísmo, su identificación de lo divino con la naturaleza. Los románticos rechazaron la

\footnotetext{
${ }^{16}$ Véase Herder, Gott, Einige Gespräche, Werke XVI, 458-64; y Schelling, Ideen zu einer Philosophie der Natur, Werke II, 20.

${ }^{17}$ Hegel, Geschichte del Philosophie, Werkausgabe XX, 165.
} 
interpretación demasiado común de Spinoza como ateo, la cual simplemente confundía natura naturans con natura naturata. En lugar de un ateo, Spinoza fue "der Gott betrunkener Mensch" porque veía todo como distintos modos de lo divino. Esta identificación de lo divino con la naturaleza parecía ser el único modo de mantener a la religión con vida en una época marcada por la ciencia. El viejo teísmo se desmoronó por la potencia de la crítica bíblica; y el deísmo se frenó ante la crítica humeana y kantiana. Tan solo el panteísmo spinoziano era invulnerable a la crítica racional o al avance científico. El eslogan deus sive natura parecía convertir en ciencia a la religión al naturalizar lo divino, pero también en religión a la ciencia al divinizar lo natural.

A pesar de los atractivos del spinozismo, los románticos pensaban que tenía problemas insuperables. La principal desventaja del sistema spinoziano era que aún estaba limitado por los anticuados dogmas de la física cartesiana. Spinoza no sólo aceptó el concepto cartesiano de materia entendido como extensión, sino que además dio por bueno el paradigma de explicación mecanicista. Pero en estos aspectos Spinoza demostró ser hijo de su tiempo. Para que el sistema spinoziano pudiera sobrevivir tenía que ser reinterpretado al hilo de los recientes avances en electricidad, magnetismo y química. Para los románticos esto sólo podía significar una cosa: la reinterpretación del spinozismo en base a su nuevo paradigma de explicación organicista.

Semejante interpretación organicista requería que se llevaran a cambio serios cambios en el sistema de Spinoza. En primer lugar, incorporaba una noción de desarrollo en su rígido y helado universo. La sustancia spinoziana era ahora nada menos que una fuerza viva, die Urkeraft aller Kräfte. Semejante sustancia ya no era estática y eterna, sino activa y temporal, experimentando un desarrollo desde lo embrionario a lo organizado, de lo indeterminado a lo determinado, de la potencia al acto. Aunque el mismo Spinoza concebía la sustancia en términos de fuerza, ${ }^{18}$ nunca entendió la fuerza en términos orgánicos en el sentido de la vis viva leibniziana. Además, su fuerza nunca actuó temporalmente, sino eternamente ya que la sustancia tan solo se relaciona con sus modos lógicamente.

En segundo lugar, la interpretación organicista introduce un elemento teológico en el sistema spinoziano. Si la sustancia divina es un organismo, entonces también ella es un fin natural cuyo objetivo consiste en desplegar su potencialidad por toda la naturaleza. Como es bien conocido, Spinoza desterró la teología de su sistema por ser antropomórfica y antropocéntrica, implicando que la naturaleza había sido

${ }^{18}$ Véase Ethica, parte I, prop. xxxiv. 
diseñada por Dios con fines humanos. Pero los románticos creían que el rechazo de la teología por parte de Spinoza era demasiado precipitado. También ellos rechazaban la vieja fisio-teología del pasado, según la cual la naturaleza no es sino un instrumento creado por Dios para servir al hombre. Pero esta era una "teología externa", que ve fines impuestos en las cosas; no es una "teología interna", que considera que los fines son inherentes a las cosas, como la idea misma o concepto del todo. Una teología interna no hace referencia necesariamente a fines humanos porque asume que el fin de una cosa se deriva de su propio concepto inherente o esencia.

En tercer lugar, la interpretación organicista también conlleva la idea de una pirámide o jerarquía en la naturaleza, la idea de "la gran cadena del ser". Spinoza había situado todos los modos en el mismo plano; una piedra, un vegetal, o un ser humano son manifestaciones iguales del infinito, que está presente al completo en todas las cosas. Sin embargo, el concepto orgánico de los románticos significaba un regreso al viejo concepto jerárquico de naturaleza. Si el universo es una fuerza viva, entonces, de la misma manera que tales fuerzas, se desarrolla por grados y fases, pasando por niveles de organización creciente. Para los románticos, el grado superior de organización y desarrollo de la fuerza divina de la naturaleza no es otro que el de la creatividad del artista. Lo que crea el artista es lo que lo divino crea a través suyo, de tal manera que las obras del artista revelan lo divino. La apoteosis del arte, que es tan característica del Frübromantik, parece absurdamente extravagante, pero se sigue inmediata e inevitablemente de tres premisas muy claras: (1) que la actividad humana es una parte de la naturaleza, de hecho es su organización y desarrollo más elevado; (2) que el arte es la forma de actividad humana más creativa, y por lo tanto la superior; y (3) que en el organismo de la naturaleza el todo está en cada una de sus partes, de tal manera que desde su propio punto de vista cada parte refleja el universo al completo. De (1) y de (3) se sigue que la actividad del artista refleja o encarna todo el universo; es el universo entero actuando a través del artista.

En cuarto lugar, la interpretación organicista también implica una nueva concepción de los atributos spinozianos, su doctrina de que lo mental y lo físico son simplemente diferentes atributos de una única sustancia indivisible. Indiscutiblemente, esta doctrina es una de las más difíciles del entender del sistema de Spinoza. Cómo la interpretemos depende de si un atributo es algo meramente subjetivo —el modo en el que el intelecto explica o entiende la sustancia- o si es algo objetivo - una propiedad de lo divino que se sigue necesariamente de su naturaleza-. Pero independientemente de cómo entendamos la doctrina 
de Spinoza, y de mucho o poco que nuestra interpretación lo acerque a los románticos, sigue habiendo un aspecto en el que los románticos la reinterpretan. A saber, conciben los atributos spinozianos en términos organicistas, como diferentes grados de organización y de desarrollo de la fuerza vital. Lo mental y lo físico no son simplemente diferentes propiedades o perspectivas de la sustancia, sino grados diferentes de organización y desarrollo de la fuerza vital.

Es justo afirmar que, al convertir el universo spinoziano en un organismo, los románticos lo reinterpretaron siguiendo los criterios leibnizianos. Los románticos fusionaron la vis viva de Leibniz con la sustancia infinita singular de Spinoza, creando un panteísmo vitalista, o un vitalismo panteísta. Si bien aceptaban el monismo de Spinoza, rechazaban su mecanicismo; si bien rechazaban el pluralismo de Leibniz, aceptaban su vitalismo, su concepto orgánico de naturaleza implícita en su dinámica. Fue con esta notable fusión de Leibniz y de Spinoza — los dos grandes metafísicos dogmáticos del siglo XVII — con la que los románticos intentarían resolver la aporia de la época post-kantiana.

\section{LOS FELICES LAZOS NUPCIALES DEL IDEALISMO Y EL REALISMO}

Debemos interpretar el intento por parte de los románticos de fusionar el idealismo con el realismo en estos términos como un spinozismo reavivado. La unión de Leibniz con Spinoza era la base para la unión de Fichte con Spinoza. Un monismo vitalista, o un vitalismo monista, parecía ser la mejor manera de preservar las verdades, y de negar los errores, del idealismo de Fichte y del realismo de Spinoza.

$\mathrm{El}$ intento por parte de los románticos de fusionar el idealismo con el realismo ante todo tiene que ser entendido como un intento de reavivar la doctrina spinoziana de los atributos. Según esta doctrina, podemos observar el universo entero, la sustancia única infinita, como pensamiento o como extensión. Lo mental y lo físico no serían ya diferentes sustancias sino simplemente diferentes atributos — propiedades o perspectivas (o ambas) — de una única cosa. Ya no existiría el problema de tener que explicar la interacción entre lo mental y lo físico porque no son ya cosas heterogéneas. La sustancia única infinita —el universo entero- permanece indivisa dado que es posible explicar cualquier cosa apelando ya sea al atributo del pensamiento o al de la extensión.

Según al menos una interpretación de esta difícil doctrina spinoziana, parece que otorga el mismo valor a las afirmaciones del idealismo que a las del realismo. Si lo interpretamos todo según el atributo del pensamiento, estaremos 
procediendo idealmente, como si todo lo que existe fuera mental o ideal; pero si lo explicamos todo según el atributo de la extensión, nuestra concepción será materialista o realista, como si todo lo que existe tan solo fuera una apariencia o manifestación de la materia. Sean cuales sean los méritos de esta interpretación, los románticos la suscribieron y consideraron que les daba pie para llevar a cabo la síntesis entre idealismo y realismo. Reinterpretaron el principio fichteano de la identidad sujeto-objeto en conformidad con Spinoza. La identidad de sujeto y objeto no está localizada en el auto-conocimiento del sujeto trascendental, sino en la sustancia única infinita, la cual permanece siendo la misma tanto si es interpretada subjetivamente (idealismo) como si es interpretada objetivamente (realismo).

Los románticos, claro está, dan un giro organicista a la doctrina spinoziana, de tal manera que la síntesis entre idealismo y realismo debe ser entendida en términos organicistas. Podemos interpretar el universo entero en términos idealistas siempre y cuando lo veamos todo de arriba a abajo y consideremos que la materia no es sino el grado más bajo de organización y desarrollo de las fuerzas vitales presentes en la mente. También podemos interpretarlo en términos realistas siempre y cuando lo veamos todo de abajo a arriba, y consideremos que la mente no es otra cosa que la organización y el desarrollo de las potencias vitales presentes de manera potencial e inherente en la materia. Si la naturaleza no es más que la mente visible, y la mente no es más que la naturaleza invisible, entonces tanto el idealismo como el realismo serían correctos. Lo importante es no privilegiar una forma o explicación por encima de la otra; ambas son perspectivas independientes e igualmente válidas de una misma realidad, de una fuerza vital.

Sería ingenuo pensar que esta síntesis de idealismo y realismo acomoda ambos puntos de vista sin coste alguno, como si todas sus afirmaciones pudieran ser aceptadas sin más. Para preservar sus aportaciones sus ilusiones deben ser negadas. Una aportación del idealismo fue pensar que la naturaleza puede ser comprendida en términos teológicos, asumir que todo puede ser explicado en términos de autoconciencia o subjetividad. La auto-conciencia es, de hecho, el fin de la naturaleza, el nivel superior de organización y desarrollo de todas sus capacidades. Pero, según los románticos, los idealistas se extraviaron cuando confundieron lo teleológico con lo ontológico; en otras palabras, si la auto-conciencia y la subjetividad es el fin de la naturaleza al completo, no se sigue que todo exista únicamente en alguna autoconciencia o sujeto. Que la subjetividad sea el propósito de las cosas no quiere decir que tan solo exista la subjetividad. Así, el principio de Fichte de que todo se deriva del ego es correcto, pero eso no debería querer decir que todo existe en 
el ego, sino únicamente que la naturaleza logra su propósito último en el ego. Los idealistas no tuvieron en cuenta una vieja, pero fundamental aportación de Aristóteles: lo que es primero en el orden de la explicación no es necesariamente lo primero en el orden del ser. Aunque la mente es el fin de la naturaleza - aunque todo sea creado en aras de ella— no se sigue que la mente sea la creadora de toda la naturaleza.

Con respecto a la perspectiva realista, está en lo correcto al pensar que hay una naturaleza que existe con independencia de la subjetividad, en asumir que la naturaleza existe al margen de, y con anterioridad a, la consciencia que los humanos tienen de la misma. Si la subjetividad es la primera en el orden de la explicación, la objetividad es la primera en el orden del ser. Es ciertamente correcto que la auto-conciencia humana es únicamente una manifestación y un desarrollo de las potencias que están implícitas, en estado embrionario y potencial en la materia. Con todo, el realista se extravía cuando piensa que la naturaleza es una cosa en sí misma indiferente a lo humano, a lo subjetivo, o ideal. Si la auto-conciencia humana es el nivel superior de organización y desarrollo de todos los poderes orgánicos de la naturaleza, entonces la naturaleza tan solo se actualizará completamente y quedará determinada a través de ella. Si, por la razón que sea, no hubiera una autoconciencia, la naturaleza no se realizaría completamente. Sin lugar a dudas seguiría existiendo, pero tan sólo en alguna forma potencial, embrionaria e indeterminada. Sería como un árbol joven que no llega a convertirse nunca en un enorme roble.

Se verá fácilmente que la acomodación del idealismo en el interior del spinozismo organicista por parte de los románticos le confiere una importancia esencialmente teleológica. Los románticos quieren que el universo no siga mostrándose indiferente hacia el ser; y evitan esta severa implicación del spinozismo volviendo a coronar al yo como culminación de la creación. Puede perfectamente ser objetado que esto no es más que una recaída en el antropomorfismo y en el antropocentrismo, los mismos vicios que Spinoza había encontrado en la teleología. Es importante que reparemos, no obstante, en que se trata en realidad de una nueva forma de antropomorfismo y antropocentrismo, uno que nunca estuvo al alcance de Spinoza. Como ya hemos visto, los románticos afirman que el blanco de la polémica spinozista era la teleología externa de la vieja físico-teología, según la cual todo existe únicamente para el hombre, como si únicamente fueran medios para sus fines. Según esta teleología los alcornoques existen porque los hombres necesitan tapones para las botellas de vino. Por el contrario, el antropomorfismo y el antropocentrismo de los románticos surgieron de una teleología interna según la cual todo lo que existe en la naturaleza es un fin en sí mismo. Los románticos 
afirmaban que es siguiendo las leyes inherentes de $s u$ desarrollo como un propósito natural prepara el terreno para que se forme la auto-conciencia humana.

Es digno de mención en este contexto que el concepto orgánico de naturaleza de los románticos implica que todo es recíprocamente un medio y un fin. Dependiendo de nuestro punto de vista, podremos ver cada parte de un organismo como un instrumento para el desarrollo del todo, o el todo como un instrumento para el desarrollo de cada parte. Quiere esto decir que es posible tanto que el hombre se desarrolle en aras de la naturaleza, como que la naturaleza se desarrolle en aras del hombre. Si los románticos subrayen tan a menudo una posibilidad antes que la otra —el aspecto más antropomórfico o antropocéntrico- es únicamente porque les preocupa mitigar el regusto amargo de la sustancia indiferente spinoziana, su completa independencia con respecto a cualquier preocupación humana. Para los románticos, esta creencia era únicamente otro de los legados problemáticos de la física mecánica, cuya desaparición podía empezar a entreverse gracias al concepto orgánico de naturaleza.

\section{RENOVANDO Y REVITALIZANDO LA EPISTEMOLOGÍA}

El concepto orgánico de naturaleza de los románticos rompió en aspectos fundamentales con la tradición epistemológica cartesiana, una tradición que había analizado el conocimiento de acuerdo con algunas de las suposiciones fundamentales de su física mecánica. Una de esas suposiciones es que el sujeto y el objeto del conocimiento, como todas las sustancias en la naturaleza, se relacionan únicamente mediante causalidad eficiente. El sujeto y el objeto son, ambos, sustancias, entidades autosuficientes e independientes, conectadas únicamente por medio de relaciones de causa y efecto. O bien el sujeto es la causa del objeto, como ocurre en el idealismo, o el objeto es la causa del sujeto, como en el realismo, o bien ambos son causa y efecto del otro, como ocurre en algunas combinaciones de realismo e idealismo. Cualquiera que sea el caso, hay únicamente una interacción causal entre el sujeto y el objeto que no altera la identidad de ambos términos. Otra suposición era el dualismo cartesiano. Dado que el mecanicismo no era capaz de explicar la res cogitans, la situaba en un dominio sui generis más allá del orden de la naturaleza, que consistía exclusivamente de res extensa. Este dualismo implicaba que el conocimiento del mundo externo tendría que consistir en dos términos muy diferentes: una representación mental y un objeto extenso. Esta correspondencia se entiende por lo general como una clase de semejanza o isomorfismo. 
Estas suposiciones, claro está, crearon dificultades insuperables a la hora de intentar explicar la posibilidad de conocimiento. Si la representación pertenece al ámbito mental y si su objeto pertenece al ámbito físico, entonces ¿cómo puede haber una correspondencia entre ellos?, ¿Cómo es posible atribuir una semejanza entre estas entidades heterogéneas? La segunda suposición del modelo cartesiano socava la primera ya que la primera supone que la correspondencia se produce por medio de una interacción causal; pero la segunda hace que sea imposible concebir relaciones causa-efecto entre lo mental y lo físico. Si bien el modelo mecánico presupone que un objeto actúa sobre otro por impacto, donde el impacto se mide por el cambio de posición en un tiempo dado, el dualismo mental-físico implica que la res cogitans no ocupa ningún espacio, por lo que es imposible concebir de qué manera una causa física tiene un efecto mental. Aunque el modelo mecánico funciona bien entre objetos extensos en el mundo natural, no afecta a objetos que no son extensos, tales como la sustancia pensante que es el locus del conocimiento. Por lo tanto el resultado neto de la concepción cartesiana de la naturaleza es que el conocimiento acaba siendo un misterio absoluto. Es imposible explicar en qué consiste la semejanza entre la representación y su objeto y, de hecho, cómo llega a producirse.

Si reemplazamos el modelo mecánico de la naturaleza por el orgánico, estos misterios de la epistemología tradicional desaparecen. En primer lugar, no hay dualismo entre lo mental y lo físico dado que ambos son grados de organización y desarrollo de la fuerza vital. Por lo tanto no hay problema a la hora de explicar la correspondencia entre entidades heterogéneas. La representación del objeto por parte del sujeto no se encuentra en un mundo diferente al del objeto sino que únicamente es el grado superior de organización y desarrollo de las fuerzas vitales en el mundo objetivo. En segundo lugar, no hay únicamente relaciones causales accidentales entre el sujeto y el objeto, sino lazos más estrechos de identidad en los que cada uno realiza su identidad únicamente en y por medio del otro. Según el modelo orgánico, todo lo que hay en la naturaleza forma parte de un todo orgánico, donde cada parte es inseparable del todo, y donde el todo es inseparable de cada una de sus partes. Ninguna parte posee una naturaleza auto-suficiente o independiente en la que conserve su identidad aparte de todo lo demás; por el contrario, cada parte refleja la identidad del todo. Como todas las partes de un todo orgánico, el sujeto y el objeto están internamente relacionados el uno con el otro de esta manera. La conciencia de un objeto que tiene un sujeto desarrolla y realiza las capacidades de un objeto, de tal manera que la conciencia del objeto no es otra cosa que la auto-realización del objeto. Ya que la creatividad artística y 
la contemplación filosófica son la organización y el desarrollo superior de todas las potencias de la naturaleza, la conciencia que tienen el artista y el filósofo de la naturaleza no es otra cosa que la auto-conciencia de la naturaleza por medio del artista y el filósofo.

El concepto orgánico de naturaleza comporta esencialmente un modelo de la conexión entre lo mental y lo físico completamente diferente del dominante en la tradición cartesiana. La conexión ya no es simplemente una conexión causal en la que cada término mantiene su identidad independiente de la interacción; por el contrario, es una conexión teleológica en el sentido de que cada término realiza su naturaleza únicamente por medio del otro. Cada término llega a ser lo que es únicamente por medio del otro, hasta el punto que únicamente llega a estar organizado, actualizado y determinado por medio del otro, de manera que sin el otro sigue siendo embrionario, potencial e indeterminado. Mientras el análisis del conocimiento permanezca anclado en el modelo causal será imposible explicar cómo es posible el conocimiento, porque la actividad ejercida por el sujeto sobre el objeto, o por el objeto sobre el sujeto, afectará a la representación del objeto, con lo que nos facilita conocimiento únicamente de cómo el sujeto afecta al objeto o de cómo el objeto afecta al sujeto, pero no nos da conocimiento del objeto mismo al margen de y con anterioridad a que se produzca la interacción. ${ }^{19}$ De ahí que el resultado inevitable a partir del modelo mecanicista de interacción fuera la idea de una cosa en sí misma incognoscible.

Los románticos estaban convencidos de que, gracias al nuevo modelo orgánico de la naturaleza, por fin habían superado la tradicional antítesis entre el idealismo y el realismo. Mantenían que el idealismo y el realismo eran perspectivas unilaterales, siendo ambas verdaderas y falsas: son verdaderas con respecto a una parte del todo, pero son falsas con respecto al todo. Si la naturaleza es un todo orgánico, no es posible decir, con el idealismo, que se encuentra en el interior de la conciencia, pero tampoco, con el realismo, que se encuentra completamente en el exterior de la conciencia. En verdad es ambas cosas y ninguna. ${ }^{20} \mathrm{El}$ todo orgánico está en el interior de la conciencia porque la conciencia es la organización y el desarrollo más elevado de todas sus potencias vitales; la conciencia que el filósofo y del artista tienen de la naturaleza es de toda la naturaleza que ha alcanzando la auto-conciencia

\footnotetext{
${ }^{19}$ Sobre este razonamiento, véase Schelling, System der gesammten Philosophie, Werke VI, 138-40, \$1. ${ }^{20}$ Véase Schelling, System der gesammten Philosophie, Werke VI, 141-5, §4; y Schelling y Hegel, Fernere Darstellungen aus dem System der Philosophie, Werke VI, 356-61; y Novalis, Allgemeine Broullion, HKA III, 382-4 (\#634), 252 (\#69), 382 (\#633), 429 (\#820).
} 
por medio de ellos, por lo que toda la naturaleza tiene su punto culminante en la creatividad artística y en la contemplación filosófica. El todo orgánico también esta en el exterior de la conciencia porque la conciencia humana es tan solo una parte de la naturaleza, la cual existe al margen de, y con anterioridad a, nosotros. Sin la humanidad la naturaleza no culmina su propósito; permanece en estado embrionario, desorganizada e indeterminada; pero de esto no se sigue que no exista. Desde esta perspectiva, así pues, queda demostrado que la idea de una cosa en sí misma es absurda. Así como es verdad que la naturaleza existe al margen de nuestra conciencia de ella, es falso que la naturaleza tenga una naturaleza completa y auto-consciente al margen de nuestra conciencia de la misma; semejante idea no sería más que una abstracción artificial de la idea de un todo orgánico.

\section{LA CUESTIÓN DE LA LIBERTAD}

Una pregunta permanece sin respuesta: ¿De qué manera lograron los románticos cuadrar su nueva metafísica con su creencia en la libertad? Parece existir un conflicto irreconciliable entre esa creencia y su panteísmo, según el cual todo ocurre necesariamente de acuerdo con las leyes de la naturaleza. En última instancia, el hecho de que estas leyes sea teleológicas a la vez que mecánicas importa poco. En este respecto, su panteísmo parece idéntico al de Spinoza; pero Spinoza era célebre por su fatalismo así como por su ateísmo. ¿De qué manera, así pues, evitaron los románticos la acusación de fatalismo? Este era un problema que inquietaba profundamente a Friedrich Schlegel, a Schelling y a Novalis, quienes lo trataron en sus clases, cuadernos y borradores desde finales de la década de 1790 hasta principios de la de $1800 .^{21}$

El concepto orgánico de naturaleza de los románticos hacía que fuera imposible para ellos aceptar la solución dada por Kant y Fichte al problema de la libertad. Pues para este concepto es fundamental la convicción en la unidad de la naturaleza, en la unicidad de lo subjetivo y lo objetivo, de lo ideal y lo real. Sin embargo, la solución de Kant y de Fichte al problema de la libertad presuponía un dualismo entre estos ámbitos. Para conservar la libertad, Kant y Fichte postularon un ámbito nouménico por encima y más allá del ámbito fenoménico

\footnotetext{
21 Véase Schelling, System der gesammten Philosophie, Werke VI, 538-70, \$\$302-17; Schlegel, Transcendentalphilosophie KA XII, 50, 52, 57, 72, 74, 86; y Novalis, Die Lehrling zu Sais, HKA I, 77, Fichte-Studien, HKA II, 154, 202, 270, y Allgemeine Broullion, HKA III 271 (\#172), 381-2 (\#633-4), 404 (\#713).
} 
de la naturaleza; mientras el ámbito nouménico se ajusta a las leyes morales impuestas por la razón, el ámbito fenoménico está gobernado por una necesidad estricta de acuerdo con las leyes de la naturaleza. Los románticos rechazaron esta solución básicamente por sus implicaciones dualistas. Cuestionaron también los fundamentos de este dualismo: que el ámbito de la naturaleza está gobernado de manera estricta por leyes mecánicas. Y si la naturaleza no obedece únicamente leyes mecánicas, entonces el problema de la libertad y la necesidad tiene que ser repensado.

Fieles a su anti-dualismo, los románticos situaron al yo en la naturaleza, e insistieron en que es un modo de la sustancia única e infinita, una parte del organismo universal. No son menos naturalistas que Spinoza: también ellos afirman que todo está en la naturaleza, y que todo lo que está en la naturaleza respeta sus leyes. Contrariamente a una cierta imagen popular del romanticismo, los románticos no admiten el capricho, la arbitrariedad o la casualidad en la naturaleza. Al contrario, mantienen que todo lo que tiene lugar en la naturaleza ocurre necesariamente, de tal manera que no podría haber sido de otro modo. Además, los románticos no cuestionan que todo lo que ocurre debe ocurrir de acuerdo con leyes mecánicas, de tal manera que para todo evento exista una causa anterior que determina su curso de acción. No difieren de Spinoza porque eximan ciertos eventos de la necesidad mecánica, sino porque someten la necesidad mecánica a leyes orgánicas superiores. No es que haya leyes orgánicas especiales que, de alguna manera, se encuentran fuera de la jurisdicción del mecanicismo; más bien es que el mecanicismo está subordinado al organicismo. Lo mecánico es simplemente un caso limitado de lo teleológico, uno que se deriva de una perspectiva parcial que únicamente tiene en consideración a las partes en sus relaciones inmediatas las unas con las otras, pero no en su relación con el todo. El mecanicismo considera lo que le ocurre a determinados eventos bajo ciertas condiciones iniciales, pero en ningún momento se pregunta por los cómos y los porqués de estas condiciones iniciales; en lugar de eso, permite que la serie de causas se precipite en un regreso ad infinitum.

Debido a su compromiso con el monismo y con el naturalismo, los románticos no podían admitir una libertad tan radical como la pretendida por Kant y Fichte. Así que ponen en cuestión la posibilidad de una libertad en los dos sentidos defendidos por Kant y Fichte. En primer lugar, el concepto kantiano de libertad como espontaneidad según el cual el yo inicia una serie causal sin que exista una determinación por parte de alguna causa previa. En segundo lugar, el concepto fichteano de autoposición, según el cual el yo se hace a sí mismo, no tiene una esencia por naturaleza. Dado que ambos conceptos excluyen cualquier 
determinación por causas naturales, presuponen el dualismo noúmeno-fenómeno que los románticos rechazan. ${ }^{22}$

Dados su naturalismo y su monismo, y dado su rechazo a la libertad trascendental, podría parecer que los románticos no admiten la libertad. ¿En qué sentido estaba dispuestos a admitir la libertad? Su programa consistía en reconciliar la libertad y la necesidad, mostrar que la verdadera libertad y necesidad no son opuestos sino que, en última instancia, son lo mismo. Contemplan esta unidad en la naturaleza divina, que es libre en el sentido spinoziano, antes que en cualquier otro lugar: actúa únicamente desde la necesidad de su propia naturaleza. Es sorprendente que mientras Schlegel y Schelling rechazan atribuir libertad a alguna parte de la naturaleza, están de acuerdo en atribuírsela a la naturaleza al completo, a la sustancia divina infinita. ${ }^{23}$ Esta sustancia es libre en el sentido de que es causa sui: auto-causada y auto-realizada. Como quiera que lo abarca todo no queda nada fuera de ella, por lo que no existen causas externas que la pongan en marcha. Por el contrario, para todo aquello que sea menor que el todo siempre habrá otras partes externas a ellas que determinan su curso de acción de acuerdo con las leyes necesarias. Es destacable, no obstante, que ni siquiera la sustancia infinita spinoziana es libre en el sentido de la espontaneidad kantiana o en el de la auto-intuición fichteana, ya que ambos conceptos asumen que el yo puede actuar de otra manera, que puede escoger una serie diferente de causas o tener una naturaleza diferente. Para Spinoza, la naturaleza divina no puede ser ni actuar de otro modo sin contradecirse a sí misma.

Aunque Schlegel y Schelling atribuyen libertad absoluta únicamente a la naturaleza como un todo, intentan evitar la implicación de fatalismo. A la vez que niegan que el yo es libre como una parte de la naturaleza, afirman que es libre en su unidad con la naturaleza como un todo. Distinguen entre dos perspectivas o puntos de vista: el yo considerado en sus relaciones con otras cosas, que es el yo en

\footnotetext{
${ }^{22}$ Esta generalización de la identificación de razón y naturaleza por parte de los románticos es especialmente evidente en el caso de Friedrich Schlegel y en el de Schelling; pero también es evidente en el caso de Schleiermacher, quien en sus Reden über die Religion identificó la religión con al punto de vista según el cual toda acción humana ocurre necesariamente. Véase KGA 1/2, 212: "die Religion athmet da, wo die Freiheit selbst wieder Natur geworden ist." En su manuscrito de 1790-2 Über die Freiheit, KGA 1/1, 217-356, Schleiermacher ya había defendido el determinismo y había atacado la concepción kantiana de libertad porque separaba al yo de la naturaleza.

23 Schlegel, Transcendentalphilosophie, KA XII, 57, 72, 74; y Schelling, System der gesammten Philosophie, Werke VI, 541-8, §305.
} 
tanto individuo, como una cosa distinta a otras; y el yo considerado en sí mismo, al margen de estas relaciones, que es el yo universal, un yo idéntico a todo lo demás. Si el yo individual cae bajo el dominio de la necesidad, el yo universal comparte la libertad divina. Su identidad no se limita a una parte del todo, donde todo está determinado por causas externas; sino que se extiende a la totalidad de todas las cosas, que actúa con libertad, de acuerdo únicamente con la necesidad de su propia naturaleza. La verdadera libertad, así pues, surge al compartir o participar en la necesidad divina, al ver que en todas mis acciones lo divino actúa a través de mi. Esta era la libertad del amor intelectual a Dios de Spinoza, la libertad que reconcilia el yo con la necesidad cuando reconoce su identidad con el universo entero.

Si bien siempre existió esta dimensión spinoziana en la reconciliación romántica de la libertad con la necesidad, sería erróneo limitarla solamente a esta dimensión. Su elemento único y característico procedía de su concepto orgánico de naturaleza. Este concepto reservaba un lugar mayor a la libertad que el sistema de Spinoza principalmente porque convertía a la humanidad en el télos de la naturaleza. "El hombre es libre", escribió Friedrich Schlegel, "porque es la más alta expresión de la naturaleza." 24 Si el yo es la organización y el desarrollo más elevado de todas las potencias de la naturaleza, entonces la naturaleza deja de ser un poder externo fuera del yo, una causa externa que le empuja a actuar. Más bien la naturaleza se convierte en parte del yo porque sus propios fines se logran a través suyo. Si el yo es la más alta expresión de la naturaleza, entonces la naturaleza se contrae hasta los límites del yo mientras el yo se extiende hasta la totalidad de la naturaleza. La reciprocidad de medios y fines significa no sólo que el yo es un medio para los fines de la naturaleza, sino también que la naturaleza es únicamente un medio para el yo. Toda la naturaleza se convierte en el cuerpo orgánico del yo. En cuanto el yo comprende su identidad con la naturaleza, pasa a considerar la determinación por parte de la naturaleza como otra forma de auto-determinación.

Si los románticos tuvieron éxito conservando algún resto de libertad en su universo orgánico, hay que decir que no fue en el sentido radical de libertad con el que muchos de ellos habían comenzado. Tuvieron que abandonar el sentido de libertad como espontaneidad y como auto-posición. Enfrentados a la dramática elección fichteana entre criticismo y dogmatismo optaron por el dogmatismo, afirmando una necesidad universal. Ciertamente se fue produciendo un notable cambio en el ethos romántico a la vez que los días de ardor revolucionario fueron

${ }^{24}$ Schlegel, Transcendentalphilosopbie, KA XII, 72. 
quedando atrás. El lema ya no era cambiar el mundo obedeciendo las demandas de la razón, sino percibir la razón en la naturaleza y la sociedad, reconciliarse uno mismo con su necesidad..$^{25}$ Moral y políticamente el matrimonio pretendido por los románticos entre Fichte y Spinoza, entre idealismo y realismo, estaba desequilibrado porque desmerecía el esfuerzo titánico de Fichte y favorecía el estoicismo beatífico de Spinoza. Como todos los matrimonios entre parejas incompatibles, alguno tiene que ceder algo. Se trata del precio que hubo que pagar por una alianza, por lo demás, notable.

Frederick C. Beiser

Syracuse University

fbeiser@syr.edu

\section{CITAS Y ABREVIATURAS}

AA Kant, Immanuel, Gesammelte Scbriften, Akademie Ausgabe, Wilhem Dilthey (ed.), Berlin, de Gruyter, 1902f. Todas las referencias a la Kritik der reinen Vernunft se refieren a la primera y segunda edición, citados como A y B respectivamente.

HKA Hardenberg, Friedrich von, Novalis Schriften, Kritische, Richard Samuwl, Hans Joachim Mähl y Gerhard Schulz (eds.), Stuttgart, Kohlhammer, 1960-88.

GSA Hölderlin, Friedrich, Sämtliche Werke, Grosse Stuttgarter Ausgabe, Friedrich Beissner (ed.), Stuttgart, Kohlhammer, 1961.

KA Schlegel, Friedrich, Kritische Friedrich Schlegel Ausgabe, Ernst Behler, Jean Jacques Anstett y Hans Eichner (eds.), Munich y Paderborn, Schöningh, 1958f.

KGA Schleiermacher, Friedrich Daniel, Kritische Gesamtausgabe, Hans-Joachim Birkner, Gerhard Ebeling, Hermann Fischer y Günter Meckenstock (eds.), Berlin, de Gruyter, 1984f.

Todas las referencias a Schelling remiten a Sämtliche Werke, K. F. A. Schelling (ed.) Stuttgart, Cotta, 1856-61. Todas las refencias a Fichte remiten a Fichtes Werke, I. Fichte (ed.), Berlin, Veit, 1845-6. Todas las referencias a Herder remiten a Sämtliche Werke, B. Suphan (ed.), Berlin, Wiedmann, 1881-1913. Todas las referencias a Hegel remiten a Werke in zwanzig Bänden, Werkausgabe, E. Moldenhauer y K. Michel (eds.), Frankfurt, Suhrkamp, 1969.

${ }^{25}$ Véase Schleiermacher, Über die Religion, KGA II/1, 232. 